\title{
Effectiveness of the 2014 European Society of Cardiology guideline on sudden cardiac death in hypertrophic cardiomyopathy: a systematic review and meta-analysis
}

\author{
Constantinos O'Mahony, 1,2,3 Mohammed Majid Akhtar, ${ }^{1,3,2}$ Zacharias Anastasiou, ${ }^{4}$ \\ Oliver P Guttmann, 1,3,2 Pieter A Vriesendorp, ${ }^{5}$ Michelle Michels, ${ }^{5}$ Damiano Magrì, ${ }^{6}$ \\ Camillo Autore, ${ }^{6}$ Adrián Fernández, ${ }^{7}$ Juan Pablo Ochoa, ${ }^{7,8,9}$ Kevin M W Leong, ${ }^{10}$ \\ Amanda M Varnava, ${ }^{10}$ Lorenzo Monserrat, ${ }^{9,11}$ Aristides Anastasakis, ${ }^{12}$ \\ Pablo Garcia-Pavia, ${ }^{3,13,14,15}$ Claudio Rapezzi, ${ }^{16}$ Elena Biagini, ${ }^{16}$ Juan Ramon Gimeno, ${ }^{3,17}$ \\ Giuseppe Limongelli, ${ }^{3,18}$ Rumana Z Omar, ${ }^{4}$ Perry M Elliott ${ }^{1,3,2}$
}

\begin{abstract}
- Additional material is published online only. To view please visit the journal online (http://dx.doi.org/10.1136/ heartjnl-2018-313700)
\end{abstract}

For numbered affiliations see end of article.

Correspondence to Dr Constantinos O'Mahony, The Inherited Cardiac Diseases Unit, Barts Heart Centre, St Bartholomew's Hospital, London EC1A 7BE, UK; c.omahony@ doctors.org.uk

Received 5 June 2018 Revised 18 September 2018 Accepted 22 September 2018 Published Online First 26 October 2018

Check for updates

(c) Author(s) (or their employer(s)) 2019. No commercial re-use. See rights and permissions. Published by BMJ.

To cite: O'Mahony $C_{\text {, }}$

Akhtar MM,

Anastasiou Z, et al. Heart

2019:105:623-631.

\section{ABSTRACT}

Objective In 2014, the European Society of Cardiology (ESC) recommended the use of a novel risk prediction model (HCM Risk-SCD) to guide use of implantable cardioverter defibrillators (ICD) for the primary prevention of sudden cardiac death $(S C D)$ in patients with hypertrophic cardiomyopathy (HCM). We sought to determine the performance of HCM Risk-SCD by conducting a systematic review and meta-analysis of articles reporting on the prevalence of SCD within 5 years of evaluation in low, intermediate and high-risk patients as defined by the 2014 guidelines (predicted risk $<4 \%, 4 \%-<6 \%$ and $\geq 6 \%$, respectively).

Methods The protocol was registered with PROSPERO (registration number: CRD42017064203). MEDLINE and manual searches for papers published from October 2014 to December 2017 were performed. Longitudinal, observational cohorts of unselected adult patients, without history of cardiac arrest were considered. The original HCM Risk-SCD development study was included a priori. Data were pooled using a random effects model.

Results Six $(0.9 \%)$ out of 653 independent publications identified by the initial search were included. The calculated 5 -year risk of SCD was reported in 7291 individuals (70\% low, 15\% intermediate; $15 \%$ high risk) with 184 (2.5\%) SCD endpoints within 5 years of baseline evaluation. Most SCD endpoints (68\%) occurred in patients with an estimated 5 -year risk of $\geq 4 \%$ who formed $30 \%$ of the total study cohort. Using the random effects method, the pooled prevalence of SCD endpoints was $1.01 \%$ (95\% Cl 0.52 to 1.61$)$ in low-risk patients, $2.43 \%$ (95\% Cl 1.23 to 3.92 ) in intermediate and $8.4 \%$ (95\% Cl 6.68 to 10.25$)$ in high-risk patients.

Conclusions This meta-analysis demonstrates that HCM Risk-SCD provides accurate risk estimations that can be used to guide ICD therapy in accordance with the 2014 ESC guidelines.

Registration number PROSPERO CRD42017064203;Preresults.

\section{INTRODUCTION}

Hypertrophic cardiomyopathy (HCM) is an inherited cardiac condition associated with ventricular arrhythmias and sudden cardiac death (SCD). ${ }^{1}{ }^{2}$ Observational data indicate that prophylactic treatment with implantable cardioverter defibrillators (ICD) can be life-saving but the selection of patients most likely to benefit remains the subject of debate. In 2014, the European Society of Cardiology (ESC) recommended a strategy based on the estimated risk of SCD derived from a validated risk prediction model, HCM Risk-SCD. ${ }^{2} 3$ Since publication of the 2014 ESC guideline, several studies have assessed the performance of the ESC algorithm in different clinical settings. The aim of this study was to systematically review the published literature and to measure the performance of the 2014 ESC guidelines by determining the observed prevalence of SCD in low, intermediate and high-risk patients as defined by the guidelines.

\section{METHODS}

\section{Protocol and registration}

The systematic review was carried out in accordance to the Preferred Reporting Items for Systematic Reviews and Meta-Analyses amendment to the Quality of Reporting of Meta-analyses statement and Cochrane Collaboration and Meta-analysis of Observational Studies in Epidemiology guidelines. The systematic review protocol was registered with the International Prospective Register of Systematic reviews (PROSPERO) in October 2017 (registration number:CRD42017064203).

\section{Eligibility criteria}

Study designs

All longitudinal observational cohort studies reporting SCD endpoints in patients with HCM were included, irrespective of setting. Studies which selectively reported on patient subgroups (ie, based on specific treatments, diagnostic tests or phenotype), abstracts, case reports, case series and cross-sectional studies were excluded. 


\section{Participants}

HCM was diagnosed in the presence of left ventricular maximal wall thickness (MWT) $\geq 15 \mathrm{~mm}$ unexplained by abnormal loading conditions ${ }^{4}$ or in accordance with published criteria for diagnosis in relatives of patients with unequivocal HCM. ${ }^{5}$ Only studies examining adult patients ( $\geq 16$ years of age) without prior ventricular fibrillation or sustained ventricular tachycardia were included.

\section{Intervention}

The intervention under investigation was risk assessment using HCM Risk-SCD (http://www.doc2do.com/hcm/webHCM. html) which uses seven parameters (definitions in online supplementary material) to estimate the risk of SCD within 5 years of clinical evaluation using the following equation ${ }^{3}$ :

$$
\hat{P}_{S C D \text { at 5years }}=1-0.998^{\exp (\text { Prognostic Index })}
$$

where Prognostic Index $=0.15939858 *$ maximal LV wall thickness $(\mathrm{mm})-0.00294271 *$ maximal LV wall thickness ${ }^{2}$ $\left(\mathrm{mm}^{2}\right)+0.0259082 *$ Left atrial diameter $(\mathrm{mm})+0.00446131 *$ LVOTg $_{\max }(\mathrm{mm} \mathrm{Hg})+0.4583082 *$ Family history of SCD $(\mathrm{Yes}=1 / \mathrm{No}=0)+0.82639195 *$ Non-sustained VT $(\mathrm{Yes}=1 / \mathrm{No}=0)$ $+0.71650361 *$ Unexplained syncope $(\mathrm{Yes}=1 / \mathrm{No}=0)-0.01799934$ *Age (years).

\section{Primary endpoint}

The primary endpoint was SCD or an equivalent event. SCD was defined as witnessed sudden death with or without documented ventricular fibrillation or death within 1 hour of new symptoms or nocturnal deaths with no antecedent history of worsening symptoms. ${ }^{6}$ Aborted SCD during follow-up and appropriate ICD shock therapy were considered equivalent to SCD. ${ }^{7-12}$ As in previous studies, ICD shocks were considered appropriate if the treated tachyarrhythmia was ventricular in origin. ${ }^{7-12}$ Antitachycardia pacing (ATP) was not considered as an equivalent to SCD. Since HCM Risk-SCD estimates the risk of sudden death within 5 years of baseline evaluation, only SCD endpoints occurring within the initial 5 years of follow-up were considered.

\section{Contact with authors}

To reduce exclusion bias and minimise missing data, study authors of eligible studies were contacted to resolve uncertainties and provide further data.

\section{Information sources}

PubMed was searched and the reference lists of reviews, letters and editorials were scrutinised for relevant material.

\section{Search strategy}

The Medline search strategy was: (hypertrophic cardiomyopathy) AND death AND ('2014/10/01'[PDat]: '2017/12/08'[PDat]). A priori the systematic review included the HCM Risk-SCD development study. ${ }^{3}$ Only articles in English were included for the analysis and relevant titles in other languages were recorded.

\section{Study records}

Data management

The initial literature search results were uploaded to EPPI-Reviewer V.4 which was used to manage the retrieved abstracts (https://eppi.ioe.ac.uk/cms/).

\section{Selection process}

The retrieved studies were independently reviewed at the title and/or abstract level for the inclusion and exclusion criteria. Full manuscripts were obtained for all reports meeting the inclusion criteria or when there was ambiguity. The full manuscripts were then reviewed to see if the inclusion criteria were satisfied. A detailed study of authors, dates and locations was used to reduce redundancy. The reviewers were not blinded to the journal titles or to the study authors or institution. Reasons for exclusion were documented.

\section{Data collection process}

Data were extracted from the full-length manuscripts and transcribed to a purpose-built relational database (Access, Microsoft). Demographic data and methodological characteristics were collected. The number of patients in each of the following risk categories, as defined by the 2014 ESC guidelines, ${ }^{2}$ was recorded:

1. Low risk (ICD not recommended): predicted 5-year risk of SCD $<4 \%$.

2. Intermediate risk (ICD may be considered): predicted 5-year risk of SCD $4 \%$ to $<6 \%$.

3. High risk (ICD should be considered): predicted 5-year risk of SCD $\geq 6 \%$.

SCD endpoints per risk group were extracted. All extracted data were verified independently by another author and disagreements were resolved by consensus. Variations in definitions of predictors and SCD endpoints were recorded.

\section{Primary outcome}

The primary outcome was the prevalence SCD endpoints within 5 years of baseline evaluation in low, intermediate and high-risk categories.

\section{Secondary outcome}

The performance measures used to validate the HCM Risk-SCD model (C-index, D-statistic, calibration slope) were extracted from all included studies.

\section{Assessing the methodological quality of the studies}

The methodological quality of the studies was evaluated using the Newcastle-Ottawa Scale (NOS). ${ }^{13}$ Each study was judged on eight items, categorised into three groups: cohort selection, the comparability of the groups and the ascertainment of outcome. Stars were awarded for each item, with a maximum score of 9 .

\section{Data analysis and synthesis}

The study characteristics are presented descriptively. The pooled observed SCD endpoint prevalence per risk group predicted by the HCM risk model was estimated. The random effects method was used to pool the SCD prevalence after stabilising the variance by transforming the proportions using the Freeman-Tukey double arcsine method. ${ }^{14}$ The fixed effects method was also used as part of a secondary analysis. The final pooled results were back-transformed using Miller's inverse transformation with the harmonic mean of the sample sizes. ${ }^{15}$ Forest plots were generated to display the prevalence and corresponding 95\% CIs for each study and the overall pooled estimates and their CIs. ${ }^{16}$ Heterogeneity between studies was assessed by performing Cochran's Q test, ${ }^{17}$ and estimating the between-study variance of the estimates using the DerSimonian and Laird method. ${ }^{18} 19$ To examine the magnitude 


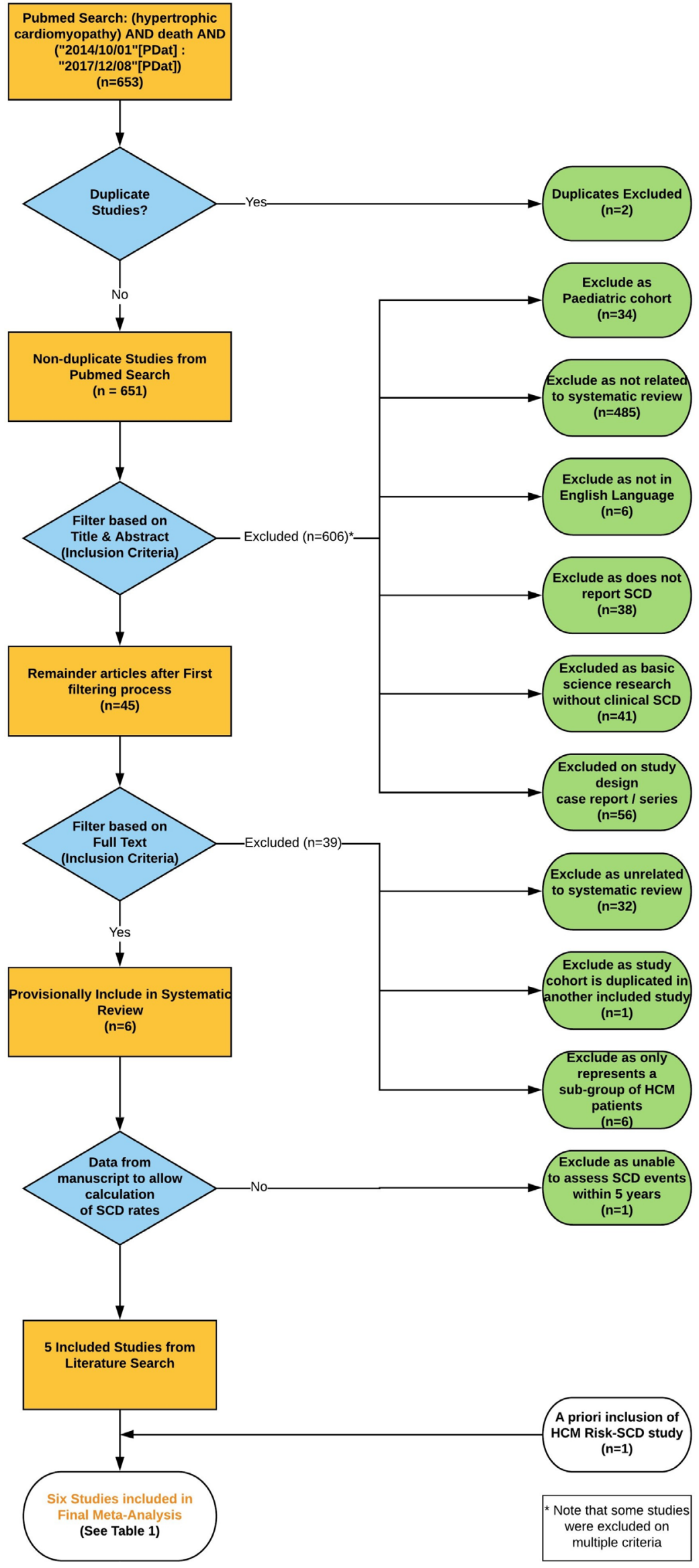

Figure 1 Study selection. The flow chart shows each step of the systematic search to identify studies reporting SCD endpoints within 5 years of baseline evaluation for low, intermediate and high-risk patients. HCM, hypertrophic cardiomyopathy; SCD, sudden cardiac death. 


\begin{tabular}{|c|c|c|c|c|c|c|}
\hline Study & $\begin{array}{l}\text { HCM Risk-SCD } \\
2014^{3}\end{array}$ & $\begin{array}{l}\text { Vriesendorp et } a^{21} \\
2015\end{array}$ & $\begin{array}{l}\text { Magrì et a }\left.\right|^{23} \\
2016\end{array}$ & $\begin{array}{l}\text { Fernández et } a l^{24} \\
2016\end{array}$ & $\begin{array}{l}\text { Leong et } a l^{26} \\
2018\end{array}$ & $\begin{array}{l}\text { EVIDENCE-HCM } \\
2018^{25}\end{array}$ \\
\hline Patients (n) & 3675 & 706 & $623^{*}$ & 502 & $288 t$ & 3703 \\
\hline Centres (n) & 6 & 2 & 5 & 1 & 1 & 14 \\
\hline Follow-up (years) & $6.6 \pm 5$ & $7.7 \pm 5.3$ & $3.7(2.2-5.1)$ & $8.6 \pm 4.3$ & $5.6 \pm 3.8$ & $7.6 \pm 6.5$ \\
\hline Annual SCD rate & 0.8 & 0.77 & 0.8 & NA & NA & 0.6 \\
\hline Age (years) & $48 \pm 17$ & $49 \pm 16$ & $49 \pm 16$ & $51 \pm 18$ & $52 \pm 16$ & $52 \pm 15$ \\
\hline MWT (mm) & $20 \pm 5$ & $20 \pm 5$ & $20 \pm 5$ & $22 \pm N A$ & $20 \pm 5$ & $20 \pm 4$ \\
\hline LVOTg $(\mathrm{mm} \mathrm{Hg})$ & $31 \pm 38$ & $48 \pm 44$ & $12(7-34)$ & $75 \pm N A$ & $28 \pm 41$ & $33 \pm 7$ \\
\hline LAd (mm) & $44 \pm 8$ & $45 \pm 8$ & $42 \pm 7$ & $46 \pm N A$ & $41 \pm 7$ & $43 \pm 8$ \\
\hline NSVT (\%) & $634(17.3)$ & $157(22.2)$ & $214(34.3)$ & $58(11.6)$ & $66(22.9)$ & $582(15.7)$ \\
\hline Syncope (\%) & $507(13.8)$ & $72(10.2)$ & $94(15.1)$ & $45(9)$ & $34(11.8)$ & $474(12.8)$ \\
\hline FHSCD (\%) & $886(24.1)$ & $141(20)$ & 71 (11.4) & $52(10.4)$ & $40(13.9)$ & $620(16.7)$ \\
\hline Validation type & Internal & External & External & External & External & External \\
\hline C-index $(95 \% \mathrm{Cl})$ & 0.7 (0.68 to 0.72$)$ & 0.69 (0.57 to 0.82$)$ & 0.69 & 0.92 (0.89 to 0.95$)$ & 0.86 & 0.7 (0.68 to 0.72$)$ \\
\hline C-index methodology & Uno & R Survival ROC & Harrell's C & ROC curves SPSS & Stata ROC & Uno \\
\hline Newcastle-Ottawa Score & 8 & 8 & 7 & 8 & 7 & 8 \\
\hline
\end{tabular}

Continuous variables are presented as mean \pm SD or median (25th-75th centiles).

*32/623 patients were also reported in HCM Risk-SCD.

†9/288 patients had cardiac arrest prior to baseline evaluation.

FHSCD, family history of sudden cardiac death; HCM, hypertrophic cardiomyopathy; LAd, left atrial diameter; LVOTg: maximal instantaneous left ventricular outflow tract gradient at rest or Valsalva; MWT, maximal wall thickness; NA, not available; NSVT, non-sustained ventricular tachycardia; ROC, receiver operating characteristic; SCD, sudden cardiac death.

of the variation between studies due to heterogeneity rather than chance, and whether it impacted the conclusions of the meta-analyses, the heterogeneity was quantified by the $\mathrm{I}^{2}$ measure. ${ }^{20}$ The estimates of the between-study variance are presented on the scale of the transformed proportions. Heterogeneity between studies was also assessed visually by inspecting the Forest plots. Stata (V.14) was used to carry out the meta-analysis.

\section{RESULTS}

A total of 653 articles fulfilled the search criteria in PubMed and six retrospective observational studies ${ }^{21-26}$ were candidates for the systematic review/meta-analysis (figure 1). One study included patients with a history of ventricular fibrillation and was included in the meta-analysis once the authors provided the required SCD endpoint data for patients without a history of cardiac arrest. ${ }^{26}$ Another study did not report SCD endpoints within 5 years of baseline evaluation; the authors were contacted but as data were not provided the study was excluded. ${ }^{22}$ The systematic review thus included five studies identified from the literature search ${ }^{21} 23-26$ and HCM Risk-SCD. ${ }^{3}$ All six studies involved subjects $\geq 16$ years of age diagnosed either with conventional or familial criteria. The included studies are summarised in table 1. Excluded studies reporting on selected HCM subgroups and non-English publications are listed in online supplementary material.

\section{Estimation of 5-year risk of SCD and SCD endpoints}

All included studies calculated the 5-year risk of SCD using the HCM Risk-SCD model equation and classified patients in the risk groups proposed by the 2014 ESC guidelines. ${ }^{32123-26}$ Uniform predictor definitions were used, except in one study ${ }^{23}$ where family history of SCD was defined as HCM-related SCD in at least one first degree or other relatives $\leq 50$ years and unexplained syncope had to be recent ( $\leq 5$ years of baseline evaluation). The same study used ATP as a surrogate of $\mathrm{SCD}^{23}$ but all other studies used the same SCD endpoint definition as stated in the Methods section. ${ }^{32124}$ Following communication with the study authors, ATP endpoints (and 32 patients reported in both HCM Risk-SCD and Magrì et $a l^{23}$ ) were excluded.

\section{SCD endpoints per SCD risk group}

The included studies reported a total of 9456 unique patients without a history of cardiac arrest but due to missing data the 5 -year risk of SCD was not available in 2165 (23\%) patients (in the original studies multiple imputation was used for these patients). The calculated 5-year risk of SCD was reported in 7291

Table 2 SCD endpoints in low, intermediate and high-risk patients within 5 years of evaluation

\begin{tabular}{|c|c|c|c|c|c|c|}
\hline Study & $\begin{array}{l}\text { HCM Risk-SCD } \\
2014^{3}\end{array}$ & $\begin{array}{l}\text { Vriesendorp et al }{ }^{21} \\
2015\end{array}$ & $\begin{array}{l}\text { Magrì et a } P^{23} \\
2016\end{array}$ & $\begin{array}{l}\text { Fernández et } a l^{24} \\
2016\end{array}$ & $\begin{array}{l}\text { Leong et } a l^{26} \\
2018\end{array}$ & $\begin{array}{l}\text { EVIDENCE-HCM } \\
2018^{25}\end{array}$ \\
\hline Low-risk patients $(<4 \%)$ & 2110 & 469 & 414 & 387 & 227 & 1524 \\
\hline SCD endpoints in low-risk patients (\%) & $24(1.1)$ & $6(1.3)$ & $9(2.2)$ & $0(0)$ & $3(1.3)$ & $16(1)$ \\
\hline Intermediate-risk patients ( $4 \%$ to $<6 \%)$ & 487 & 113 & 99 & 39 & 29 & 326 \\
\hline SCD endpoints in intermediate-risk patients (\%) & $17(3.5)$ & $6(5.3)$ & $2(2)$ & $2(5.1)$ & $0(0)$ & $5(1.5)$ \\
\hline High-risk patients (>6\%) & 469 & 124 & 78 & 76 & 23 & 297 \\
\hline SCD endpoints in high-risk patients (\%) & $43(9.2)$ & $8(6.5)$ & $6(7.7)$ & $12(15.8)$ & $2(8.7)$ & $23(7.7)$ \\
\hline
\end{tabular}

HCM, hypertrophic cardiomyopathy; $S C D$, sudden cardiac death. 


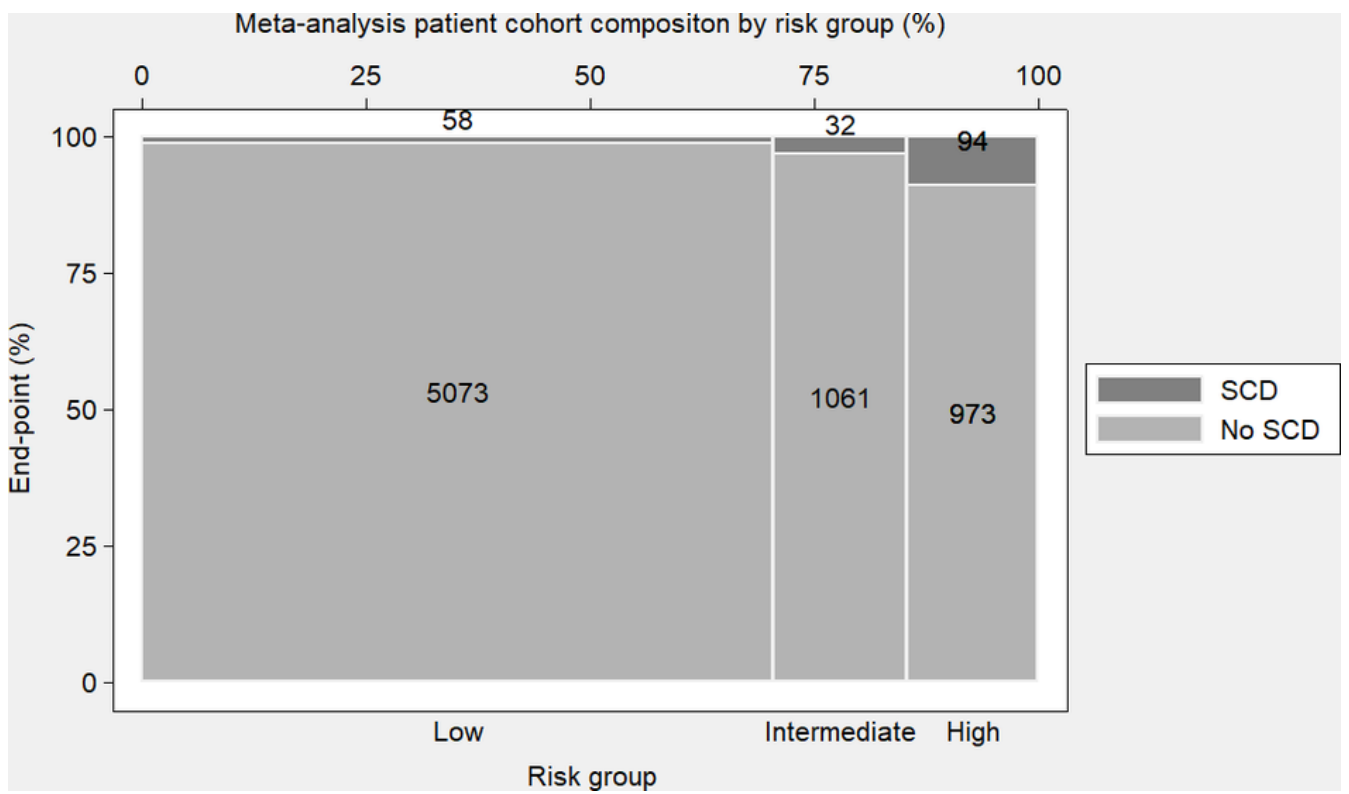

Figure 2 SCD endpoints versus SCD risk category. The mosaic plot illustrates SCD endpoints for each SCD risk category in the 7291 subjects reported in the six included studies of the meta-analysis. The heights of the elements in each stacked bar indicate the percentage of those without SCD endpoints. The width of each stacked bar is proportional to the number of observations in each risk group; the areas of the elements also indicate absolute counts (also included in the figure). SCD, sudden cardiac death.

individuals (5131 (70\%) low risk; 1093 (15\%) intermediate risk; 1067 (15\%) high risk) with 184 (2.5\%) SCD endpoints within 5 years of baseline evaluation. SCD endpoints per risk group are shown in table 2 and figure 2. High-risk patients contributed $51 \%$ of SCD endpoints; $68 \%$ of all SCD endpoints occurred in patients with an estimated 5 -year risk of $\geq 4 \%$ who formed $30 \%$ of the total study cohort.

\section{Pooled SCD endpoint prevalence per 2014 ESC risk group}

Using the random effects method, the pooled prevalence of SCD endpoints was $1.0 \%$ (95\% CI 0.52 to 1.61 ) in low-risk patients, 2.43 (95\% CI 1.23 to 3.92 ) in intermediate-risk patients and 8.39 (95\% CI 6.68 to 10.25 ) in high-risk patients (figure 3). Moderate heterogeneity was observed for the low-risk group with an $\mathrm{I}^{2}$ value of about $65 \%$. Results were similar when a fixed effects method was used and the pooled prevalence of SCD endpoints was $1.01 \%$ (95\% CI 0.74 to 1.32 ) in low-risk patients, 2.39 (95\% CI 1.46 to 3.48 ) in intermediate-risk patients and 8.38 (95\% CI 6.73 to 10.19 ) in high-risk patients.

\section{Validation measures}

The C-index, calculated with a variety of methods, ranged from 0.69 to 0.92 as shown in table 1 . Calibration slope and D-statistic were only reported in HCM Risk-SCD (calibration slope: $0.91,95 \%$ CI 0.74 to 1.08 ; D-statistic: $1.07,95 \% \mathrm{CI} 0.81$ to $1.32)^{3}$ and EVIDENCE-HCM (calibration slope: 1.02, 95\% CI 0.93 to 1.12 ; D-statistic: $1.17,95 \%$ CI 1.05 to 1.29$){ }^{25}$ The intercept of the calibration slopes was not reported.

\section{Methodological quality of included studies}

NOS ranged from 7 to 8 stars, out of a maximum of 9 (table 1). The detailed breakdown of NOS is shown in the online supplementary material.

\section{DISCUSSION}

This study analysed data from more than 7000 unselected patients with HCM from Europe, Asia, Middle East, North and
South America, and the main findings support the 2014 ESC guideline recommendations for the primary prevention of SCD. Application of these guidelines in clinical practice facilitates targeted management of the subgroup of patients in whom ICD has the greatest potential to prevent SCD and allows reassurance for the large majority of patients who are at very low risk.

The effectiveness of ICD in preventing SCD in HCM is well established but frequent device complications ${ }^{27}$ make risk stratification a crucial step in the management of all patients. Historically, SCD risk has been estimated by evaluating clinical parameters which reflect the severity of the underlying myocardial disease. This assessment is then used to guide clinical decision-making with respect to prophylactic ICD implantation. The first risk stratification strategy jointly proposed by the ESC and the American College of Cardiology (ACC) in 2003 was an algorithm based on consensus and limited contemporaneous data, and relied on a simple phenotypic assessment to provide a qualitative evaluation of risk. ${ }^{28} \mathrm{~A}$ similar approach is currently recommended by the ACC and American Heart Association. ${ }^{1}$ The major limitation of these approaches is the dependence on relative rather than absolute risk estimates to guide treatment. Furthermore, the phenotypic assessment is relatively crude in that all major risk factors are considered in a dichotomous manner which can give rise to obvious clinical dilemmas; for example, it is improbable that an MWT of $29 \mathrm{~mm}$ confers a significantly different risk than $30 \mathrm{~mm}$. To overcome these and other limitations, ${ }^{29}$ the 2014 ESC guidelines recommend a risk prediction model that provides individualised, quantitative prognostic risk estimates using a more enhanced phenotypic assessment based of seven clinical parameters previously shown to be associated with SCD. ${ }^{23}$

This meta-analysis summarises all available published data relevant to the 2014 ESC guideline performance for SCD prevention. Patients in the prespecified high-risk and low-risk categories had the highest and lowest prevalences of SCD endpoints within 5 years of baseline evaluation, respectively, and pooled prevalences are concordant with the predicted SCD risk. These 


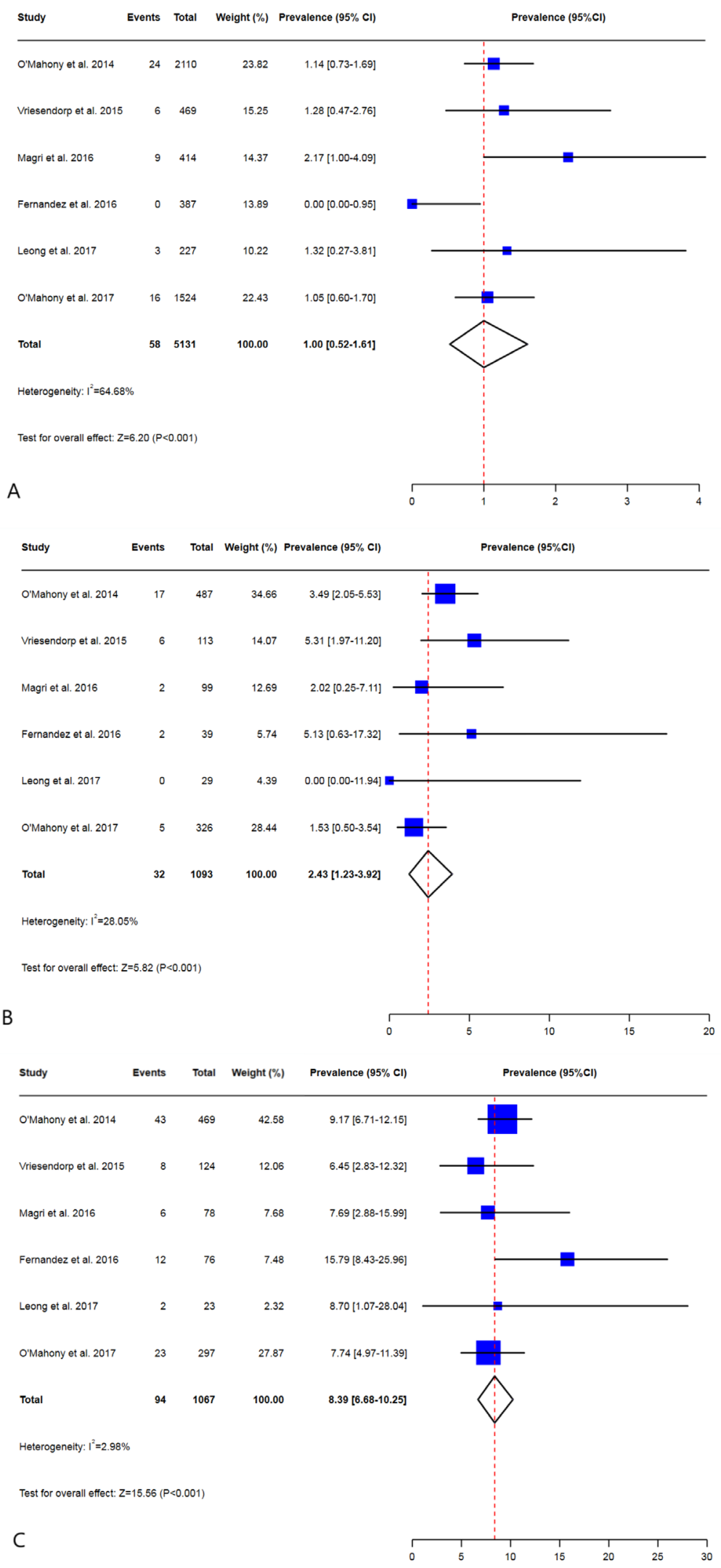

Figure 3 Prevalence of sudden cardiac death (SCD) endpoints within 5 years of baseline evaluation. The Forest plots show the prevalence (\%) and $95 \% \mathrm{Cls}$ with the random effects method for low (A), intermediate (B) and high-risk (C) patients. Patients with 5 -year risk $<4 \%$ are considered low risk and require systematic reassessment; intermediate-risk patients (5-year risk $4 \%$ to $<6 \%$ ) may be considered for an implantable cardioverter defibrillator (ICD) depending on comorbidities; high-risk patients (5-year risk $\geq 6 \%$ ) should be considered for an ICD. The between-study variance was estimated on the scale of the transformed values of the proportions. 
findings support the ESC recommendation that patients with 5 -year risk $<4 \%$ should be conservatively treated while those with an estimated 5 -year risk $\geq 6 \%$ should be considered for an ICD. ${ }^{2}$ The intermediate risk group had a relatively low prevalence of SCD endpoints and the meta-analysis suggests that the risk in these patients is overestimated. However, since more than two-thirds of SCD endpoints occur in intermediate and high-risk patients, the pragmatic approach is to continue to target these patients for a primary prevention ICD.

All included studies examined the prognostic accuracy of HCM Risk-SCD using the C-index which is a measure of how well the model discriminates between patients with and without SCD. ${ }^{30}{ }^{31}$ The reported C-indices consistently show that the model has good discrimination. Differences in the statistical methods used to derive the C-indices did not allow further exploration by pooling the data using meta-analysis and other validation measures were not consistently reported.

The included patient cohorts came from diverse geographic locations and healthcare settings but had similar clinical characteristics and annual SCD rates. Most studies had a mean follow-up period of $>5$ years which is critical in examining the performance of the HCM Risk-SCD tool. Only patients with complete data to calculate the 5-year risk of SCD were included. Some patients were inevitably lost to follow-up within the first 5 years of clinical evaluation which may lead to an underestimation of SCD endpoint prevalence. Examining the observed 5 -year SCD rates rather than the prevalence of SCD within 5 years would be more informative but these were not uniformly published.

Of the six studies identified by the literature search, only one was excluded as the number of SCD endpoints within 5 years of baseline evaluation was not provided. ${ }^{22}$ This study examined 1629 patients from two North American tertiary referral centres and suggested that the 2014 ESC guideline was unreliable as most SCD endpoints occurred in low-risk patients. These findings may be explained by an unaccounted change in risk profile beyond 5 years of baseline evaluation and by underestimation of risk due to missing data which are not reported. ${ }^{22}$ Inclusion of this study is unlikely to have altered the results of this meta-analysis as the published prevalence of SCD endpoints during long-term follow-up ( $>5$ years) was concordant with the predicted 5-year risk of SCD (SCD endpoint prevalence of 3.8\% in low-risk and $12 \%$ in high-risk patients). ${ }^{22}$

Some studies were excluded from the systematic review because they reported on patient subgroups selected by specific therapeutic or diagnostic modalities. Four studies reported on ATP and appropriate ICD shocks in a small number of primary prevention ICD recipients, often without reporting events within 5 years. ${ }^{32-35}$ Two of these studies-which included a total of 154 patients-demonstrated that events were most prevalent in high-risk patients. ${ }^{32}{ }^{34}$ Another study of 52 ICD recipients reported a higher mean estimated 5 -year risk in patients who had therapies but a breakdown of events per ESC risk group was not provided. ${ }^{35}$ Rigopoulos et al investigated 32 ICD recipients who underwent percutaneous transluminal septal myocardial ablation and found that postprocedural appropriate ICD shocks occurred in patients with a high preprocedural risk. ${ }^{33}$ In another study of 844 patients who underwent percutaneous transluminal septal myocardial ablation, there was good agreement between observed and predicted SCD endpoints within 5 years of baseline evaluation. ${ }^{36}$ Finally, in a study of 117 patients who underwent a cardiac MRI, high and intermediate-risk patients had the highest prevalence of endpoints during long-term follow-up. ${ }^{37}$ Reports excluded due to publication in non-English language were primarily reviews and thus unlikely to have an impact on the findings of the meta-analysis.

The meta-analysis also demonstrates the limitations of this approach and highlights the need for improvement. SCD endpoints in low-risk patients are the most obvious problem for individual patients and on the population level. This is not unique to HCM and it is well recognised that subgroups with the highest case fatality rates have the lowest population attributable risk and vice versa. ${ }^{38}$ Even though some events in the low-risk patients may relate to a change in clinical risk profile during follow-up which is not taken into consideration in this analysis, there is a clear need for improvement. Overestimation of risk in intermediate-risk patients should also be addressed. To improve risk prediction, the incremental value of other predictors needs to be systematically examined in appropriately powered cohort studies. Candidate predictors for future models include late gadolinium enhancement, ${ }^{37} 39$ mutations in sarcomeric protein genes $^{4041}$ and exercise testing-derived variables such as ventilatory efficiency $\left(\mathrm{VE} / \mathrm{VCO}_{2}\right)$ slope and abnormal blood pressure response to exercise. ${ }^{23}{ }^{24}$ Future studies should first consider new predictors which are readily available at minimal cost and burden to the patient. Expensive and difficult to obtain predictors should then be examined for their incremental value. ${ }^{42}$

\section{CONCLUSION}

This meta-analysis examined SCD endpoints in more than 7000 patients with HCM from around the world and the findings support the 2014 ESC guidelines on SCD. It demonstrates that low-risk patients can be treated conservatively as the prevalence of SCD endpoints within 5 years of evaluation is very low. The high prevalence of SCD endpoints in high-risk patients means that these patients should be considered for an ICD. The risk of SCD is overestimated in intermediate-risk patients, but as more than two-thirds of SCD endpoints occur in intermediate and high-risk patients, the pragmatic approach is to continue to target both these subgroups for a primary prevention ICD.

\section{Key messages}

What is already known on this subject?

- Hypertrophic cardiomyopathy (HCM) is an inherited cardiac condition associated with ventricular arrhythmias and sudden cardiac death (SCD). In 2014, the European Society of Cardiology (ESC) recommended a strategy based on the estimated risk of $S C D$ derived from a validated risk prediction model, HCM Risk-SCD.

\section{What might this study add?}

- This meta-analysis of data from six studies involving 7291 patients with $\mathrm{HCM}$ demonstrates that in most patients the observed prevalence of sudden death within 5 years of evaluation is concordant with the predicted risk.

\section{How might this impact on clinical practice?}

- The results support the risk stratification strategy proposed by the 2014 ESC guidelines which uses a risk prediction model (HCM Risk-SCD) to estimate the risk of SCD within 5 years of evaluation.

\section{Author affiliations}

${ }^{1}$ The Inherited Cardiac Diseases Unit, Barts Heart Centre, St Bartholomew's Hospital, London, UK

${ }^{2}$ UCL Centre for Heart Muscle Disease, Institute of Cardiovascular Science, University College London, London, UK 
${ }^{3}$ European Reference Network for Rare and Low Prevalence Complex Diseases of the Heart (ERN GUARD-HEART)

${ }^{4}$ Department of Statistical Science, University College London, London, UK ${ }^{5}$ Department of Cardiology, Thoraxcenter, Erasmus Medical Center, Rotterdam, The Netherlands

${ }^{6}$ Clinical and Molecular Medicine, University 'La Sapienza', Rome, Italy

${ }^{7}$ Department of Cardiology, Favaloro Foundation University Hospital, Institute of Cardiology and Cardiovascular Surgery, Buenos Aires, Argentina

${ }^{8}$ GRINCAR (Cardiovascular Research Group), University of A Coruña, A Coruña, Spain

${ }^{9}$ Scientific Department, Health In Code, A Coruña, Spain

${ }^{10}$ Imperial College Healthcare NHS Trust, London, UK

${ }^{11}$ Cardiology Department and Research Unit, A Coruña University Hospital, Galician Health Service, A Coruña, Spain

${ }^{12}$ Unit of Inherited Cardiovascular Diseases, First Department of Cardiology, University of Athens, Athens, Greece

${ }^{13}$ Heart Failure and Inherited Cardiac Diseases Unit, Department of Cardiology, Hospital Universitario Puerta de Hierro Majadahonda, Madrid, Spain

${ }^{14}$ University Francisco de Vitoria (UFV), Madrid, Spain

${ }^{15}$ Centro de Investigacion Biomedica en Red en Enfermedades Cardiovasculares (CIBERCV), Madrid, Spain

${ }^{16}$ Department of Specialised, Experimental and Diagnostic Medicine, Institute of Cardiology, University of Bologna, Bologna, Italy

${ }^{17}$ Cardiac Department, University Hospital Virgen Arrixaca, La Alberca (Murcia), Spain

${ }^{18}$ Monaldi Hospital, Second University of Naples, Naples, Italy

Contributors COM and PME designed the study and drafted the manuscript. The search strategy was developed by COM. Two independent reviewers (OPG and MMA) performed the literature search and checked the eligibility of each study. Disagreement between the two reviewing authors was resolved by a third author (COM). Data were extracted by COM and independently verified by MMA. Postpublication data required for the completion of the systematic review/metaanalysis were provided by PAV, MM, DM, CA, AF, JPO, KMWL and AMV. RZO and ZA provided statistical expertise. The quality of the included studies was assessed by two reviewers (MMA, COM). PME is the guarantor. All authors read, critically appraised, provided feedback and approved the final manuscript.

Funding This work was undertaken at University College London Hospitals/ University College London and St Bartholomew's Hospital who received a proportion of funding from the UK Department of Health's National Institute for Health Research Biomedical Research Centres funding scheme. OPG received research support from the British Heart Foundation (FS/12/86/29841) and the National Institute for Health Research University College London Hospitals Biomedical Research Centre. The present work was partially supported by a research grant from 'Sapienza' University of Rome to DM. KMWL was supported by a British Heart Foundation Grant (PG/15/20/31339) and The Dan Bagshaw Memorial Trust Fund. LM received a Carlos III Health Institute grant, Madrid, Spain (PI11/0260). GL received support from a Progetto ministeriale (MIUR), Italy (PRIN 2008). PGP was supported by Institutode Salud Carlos III (grants PI14/0967 and RD012/0042/0066) through the Plan Estatal de I+D+I 2013-2016-European Regional Development Fund (FEDER): 'A way of making Europe'. JRG received support from the Cardiovascular Research Network (RIC) from the Carlos III Health Institute, Madrid, Spain (RD12/0042/0049)

Competing interests MMA reports being supported by an unrestricted educational grant from Sanofi Genzyme, outside the submitted work. LM is an employee and a stakeholder of Health in Code SL. All other authors have nothing to disclose.

Patient consent Not required.

Provenance and peer review Not commissioned; externally peer reviewed.

Data sharing statement Not applicable.

\section{REFERENCES}

1 Gersh BJ, Maron BJ, Bonow RO, et al. 2011 ACCF/AHA guideline for the diagnosis and treatment of hypertrophic cardiomyopathy: executive summary: a report of the American College of Cardiology Foundation/American Heart Association Task Force on Practice Guidelines. Circulation 2011;124:2761-96.

2 Elliott PM, Anastasakis A, Borger MA, et al. 2014 ESC Guidelines on diagnosis and management of hypertrophic cardiomyopathy: the Task Force for the Diagnosis and Management of Hypertrophic Cardiomyopathy of the European Society of Cardiology (ESC). Eur Heart J 2014:35:2733-79.

3 O'Mahony C, Jichi F, Pavlou M, et al. A novel clinical risk prediction model for sudden cardiac death in hypertrophic cardiomyopathy (HCM risk-SCD). Eur Heart J 2014:35:2010-20.

4 Elliott P, Andersson B, Arbustini E, et al. Classification of the cardiomyopathies: a position statement from the European Society Of Cardiology Working Group on Myocardial and Pericardial Diseases. Eur Heart J 2008;29:270-6.
5 McKenna WJ, Spirito P, Desnos M, et al. Experience from clinical genetics in hypertrophic cardiomyopathy: proposal for new diagnostic criteria in adult members of affected families. Heart 1997;77:130-2.

6 Elliott PM, Poloniecki J, Dickie S, et al. Sudden death in hypertrophic cardiomyopathy: identification of high risk patients. J Am Coll Cardiol 2000;36:2212-8

7 Olivotto I, Gistri R, Petrone P, et al. Maximum left ventricular thickness and risk of sudden death in patients with hypertrophic cardiomyopathy. J Am Coll Cardiol 2003;41:315-21.

8 Monserrat L, Elliott PM, Gimeno JR, et al. Non-sustained ventricular tachycardia in hypertrophic cardiomyopathy: an independent marker of sudden death risk in young patients. J Am Coll Cardiol 2003;42:873-9.

9 Maron MS, Olivotto I, Betocchi S, et al. Effect of left ventricular outflow tract obstruction on clinical outcome in hypertrophic cardiomyopathy. N Eng/ J Med 2003;348:295-303.

10 Adabag AS, Casey SA, Kuskowski MA, et al. Spectrum and prognostic significance of arrhythmias on ambulatory Holter electrocardiogram in hypertrophic cardiomyopathy. J Am Coll Cardiol 2005:45:697-704.

11 Gimeno JR, Tomé-Esteban M, Lofiego C, et al. Exercise-induced ventricular arrhythmias and risk of sudden cardiac death in patients with hypertrophic cardiomyopathy. Eur Heart J 2009;30:2599-605.

12 Efthimiadis GK, Parcharidou DG, Giannakoulas G, et al. Left ventricular outflow tract obstruction as a risk factor for sudden cardiac death in hypertrophic cardiomyopathy. Am J Cardiol 2009; 104:695-9.

13 Wells GA, Shea B, O'Connell D, et al, 2017. The Newcastle-Ottawa Scale (NOS) for assessing the quality if nonrandomized studies in meta-analyses http://www ohri ca/ programs/clinical_epidemiology/oxford htm (accessed 25 Jun 2017).

14 Freeman MF, Tukey JW. Transformations related to the angular and the square root. The Annals of Mathematical Statistics 1950:21:607-11.

15 Miller JJ. The inverse of the freeman - tukey double arcsine transformation. Am Stat 1978;32:138.

16 Higgins JP, Thompson SG, Spiegelhalter DJ. A re-evaluation of random-effects metaanalysis. J R Stat Soc Ser A Stat Soc 2009:172:137-59.

17 Cochran WG. The combination of estimates from different experiments. Biometrics 1954;10:101-29.

18 Higgins JP, Thompson SG, Deeks JJ, et al. Measuring inconsistency in meta-analyses. BMJ 2003:327:557-60.

19 DerSimonian R, Laird N. Meta-analysis in clinical trials. Control Clin Trials 1986;7:177-88.

20 Higgins JP, Thompson SG. Quantifying heterogeneity in a meta-analysis. Stat Med 2002;21:1539-58.

21 Vriesendorp PA, Schinkel AF, Liebregts M, et al. Validation of the 2014 European Society of Cardiology guidelines risk prediction model for the primary prevention of sudden cardiac death in hypertrophic cardiomyopathy. Circ Arrhythm Electrophysiol 2015;8:829-35.

22 Maron BJ, Casey SA, Chan RH, et al. Independent assessment of the european society of cardiology sudden death risk model for hypertrophic cardiomyopathy. Am I Cardiol 2015;116:757-64.

23 Magrì D, Limongelli G, Re F, et al. Cardiopulmonary exercise test and sudden cardiac death risk in hypertrophic cardiomyopathy. Heart 2016;102:602-9.

24 Fernández A, Quiroga A, Ochoa JP, et al. Validation of the 2014 european society of cardiology sudden cardiac death risk prediction model in hypertrophic cardiomyopathy in a reference center in South America. Am J Cardiol 2016;118:121-6.

25 O'Mahony C, Jichi F, Ommen SR, et al. International External Validation Study of the 2014 European Society of Cardiology Guidelines on Sudden Cardiac Death Prevention in Hypertrophic Cardiomyopathy (EVIDENCE-HCM). Circulation 2018:137.

26 Leong KMW, Chow JJ, Ng FS, et al. Comparison of the Prognostic Usefulness of the European Society of Cardiology and American Heart Association/American College of Cardiology Foundation Risk Stratification Systems for Patients With Hypertrophic Cardiomyopathy. Am J Cardiol 2018;121:349-55.

27 Wang N, Xie A, Tjahjono R, et al. Implantable cardioverter defibrillator therapy in hypertrophic cardiomyopathy: an updated systematic review and meta-analysis of outcomes and complications. Ann Cardiothorac Surg 2017;6:298-306.

28 Maron BJ, McKenna WJ, Danielson GK, et al. American college of cardiology/ european society of cardiology clinical expert consensus document on hypertrophic cardiomyopathy. a report of the american college of cardiology foundation task force on clinical expert consensus documents and the european society of cardiology committee for practice guidelines. Eur Heart J 2003;24:1965-91.

29 O'Mahony C, Tome-Esteban M, Lambiase PD, et al. A validation study of the 2003 American College of Cardiology/European Society of Cardiology and 2011 American College of Cardiology Foundation/American Heart Association risk stratification and treatment algorithms for sudden cardiac death in patients with hypertrophic cardiomyopathy. Heart 2013;99:534-41.

30 Royston P, Sauerbrei W. A new measure of prognostic separation in survival data. Stat Med 2004;23:723-48.

31 Gönen M, Heller G. Concordance probability and discriminatory power in proportional hazards regression. Biometrika 2005;92:965-70. 
32 Ruiz-Salas A, García-Pinilla JM, Cabrera-Bueno F, et al. Comparison of the new risk prediction model (HCM Risk-SCD) and classic risk factors for sudden death in patients with hypertrophic cardiomyopathy and defibrillator. Europace 2016;18.

33 Rigopoulos AG, Daci S, Pfeiffer B, et al. Low occurrence of ventricular arrhythmias after alcohol septal ablation in high-risk patients with hypertrophic obstructive cardiomyopathy. Clin Res Cardiol 2016;105:953-61.

34 Thavikulwat AC, Tomson TT, Knight BP, et al. Appropriate implantable defibrillator therapy in adults with hypertrophic cardiomyopathy. I Cardiovasc Electrophysiol 2016;27:953-60.

35 Sugrue A, Killu AM, DeSimone CV, et al. Utility of T-wave amplitude as a non-invasive risk marker of sudden cardiac death in hypertrophic cardiomyopathy. Open Heart 2017; 4:e000561.

36 Liebregts M, Faber L, Jensen MK, et al. Validation of the HCM Risk-SCD model in patients with hypertrophic cardiomyopathy following alcohol septal ablation. Europace 2018;20:f198-f203.
37 Doesch C, Tülümen E, Akin I, et al. Incremental benefit of late gadolinium cardiac magnetic resonance imaging for risk stratification in patients with hypertrophic cardiomyopathy. Sci Rep 2017;7:6336.

38 Myerburg RJ, Interian A, Mitrani RM, et al. Frequency of sudden cardiac death and profiles of risk. Am J Cardiol 1997;80:10F-19.

39 Briasoulis A, Mallikethi-Reddy S, Palla M, et al. Myocardial fibrosis on cardiac magnetic resonance and cardiac outcomes in hypertrophic cardiomyopathy: a metaanalysis. Heart 2015;101:1406-11.

40 Lopes LR, Syrris P, Guttmann OP, et al. Novel genotype-phenotype associations demonstrated by high-throughput sequencing in patients with hypertrophic cardiomyopathy. Heart 2015;101:294-301.

41 van Velzen HG, Vriesendorp PA, Oldenburg RA, et al. Value of genetic testing for the prediction of long-term outcome in patients with hypertrophic cardiomyopathy. Am J Cardiol 2016;118:881-7.

42 Steyerberg EW. Clinical prediction models. A practical approach to development, validation and updating. New York: Springer Science+Business Media, 2009. 